\section{Mulheres vítimas de homicídio em Recife, Pernambuco, Brasil, 2009/2010: um estudo descritivo}

\author{
Female homicide victims in Recife, Pernambuco \\ State, Brazil, 2009-2010: a descriptive study \\ Mujeres víctimas de homicidios en Recife, \\ estado de Pernambuco, Brasil, 2009/2010: \\ un estudio descriptivo
}

Maria Arleide da Silva 1 José Eulálio Cabral Filho 1 Melania Maria Ramos Amorim 1 Gilliatt Hanois Falbo Neto 1

\section{Resumo}

This study investigated the epidemiological profile of female homicide victims in Recife, Pernambuco State, Northeast Brazil. An observational descriptive and prospective study included all homicides from March 2009 to February 2010 with female victims from Recife, 10 to 49 years of age. A questionnaire was used to record socioeconomic, demographic, and biological risk factors. Relatives of the victims were interviewed, and data were collected from death certificates. We identified 60 homicides during the study period. Most victims were adult women with brown skin color and low schooling and low income. Other characteristics included smoking in 39.7\%, alcohol and illicit drugs in $48.3 \%$ and $24.1 \%$, respectively, and physical and/or sexual violence in the 12 months prior to the murder in 29.3\%. Firearms were used in $69 \%$ of these homicides.

Homicide; Violence; Women
Este estudo investigou o perfil epidemiológico de mulheres vítimas de homicídio em Recife, Pernambuco, Nordeste do Brasil. Realizou-se um estudo observacional, descritivo e prospectivo, incluindo todas as mulheres em idade de 10-49 anos, vítimas de homicídio e residentes em Recife, no período de março de 2009 a fevereiro de 2010. O instrumento utilizado foi um questionário que pesquisou as características socioeconômicas, demográficas, biológicas e de risco. Entrevistaramse os familiares das vítimas e coletaram-se dados da Declaração de Óbito. Identificaram-se 60 homicídios no período do estudo. A maioria das vítimas era adulta, parda, com baixa escolaridade e baixa renda; 39,7\% eram tabagistas; o uso de álcool e de drogas ilícitas correspondeu a 48,3\% e $24,1 \%$, respectivamente; $e$ 29,3\% sofreram violência física e/ou sexual nos 12 meses anteriores ao homicídio. As armas de fogo foram utilizadas em $69 \%$ dos casos.

Homicídio; Violência; Mulheres 
O homicídio é uma causa de morte crescente na população feminina. Sua ocorrência não se restringe ao padrão de costumes de uma sociedade, comunidade ou religião, acontecendo em todo o mundo e por razões semelhantes 1,2 .

A perpetuação das relações de poder, decorrentes do lugar ocupado culturalmente pelo sexo masculino nas relações de gênero, contribui para a utilização da violência como instrumento de submissão feminina e independe das questões raciais e/ou étnicas 3 . No Brasil, a crescente taxa de homicídios vitimando mulheres tem influenciado os indicadores de saúde, como a mortalidade materna e os anos potenciais de vida perdidos (APVP) 4,5.

A tendência de homicídios na população feminina indicou duas faixas etárias no ano 2000, no Estado de São Paulo: 20-24 anos (5:100 mil habitantes) e 35-39 anos (3:100 mil habitantes), ambas muito próximas ao coeficiente do Brasil, que foi de 5,03:100 mil habitantes nessa população 6 . O Estado de Pernambuco, nos anos 2003/2007, ocupou o segundo lugar, entre os estados brasileiros, com coeficiente de morte de mulheres por homicídios de 6,63:100 mil habitantes. Sua capital, Recife, ocupa o segundo posto entre as capitais brasileiras de maior risco para esse tipo de causa externa de morte 7,8 .

O ônus social da violência cresce sobremaneira, pelos danos e sequelas emocionais e/ou físicos, vitimizando indivíduos e coletividade. $\mathrm{O}$ custo financeiro para o setor saúde é elevado, em virtude dos gastos com a assistência à morbidade, mortalidade e comorbidades, bem como com a assistência social e judiciária aos agressores e suas vítimas 9,10 .

O presente estudo teve por objetivo descrever o perfil epidemiológico de mulheres vítimas de homicídios na cidade do Recife.

\section{Método}

Realizou-se estudo descritivo, observacional, do tipo série de casos, prospectivo, no período de março de 2009 a fevereiro de 2010, para descrever o perfil epidemiológico e características individuais de risco das mulheres vítimas de homicídio. Investigaram-se aquelas com idade entre 10 e 49 anos, residentes na cidade do Recife, submetidas a necropsia na unidade do Instituto Médico Legal (IML), único serviço desta natureza na cidade. O estudo foi aprovado pelo Comitê de Ética em Pesquisa do Instituto de Medicina Integral Prof. Fernando Figueira (IMIP).

No triênio 2002/2004, a cidade do Recife ocupou o primeiro lugar em taxa acumulada de óbitos por homicídio (198,3:100 mil habitantes) $11 \mathrm{e}$, no ano 2008, apresentou a segunda maior taxa do país por esta causa externa de morte $(61,0: 100$ mil habitantes) 8 .

O instrumento utilizado para a coleta dos dados foi um questionário com perguntas fechadas, incluindo algumas variáveis da Declaração de Óbito (DO): idade, cor, instrumento utilizado para a violência letal. Para caracterização socioeconômica, demográfica e biológica das vítimas, foram investigadas as variáveis: estado civil, escolaridade, ocupação, religião, prática religiosa, renda mensal da vítima, nascimento de filho no último ano e gravidez por ocasião do homicídio. As variáveis individuais de risco foram: tabagismo, uso de bebida alcoólica, uso de drogas, envolvimento com o tráfico de drogas, uso de arma, passagem pela polícia, exposição a violência física e/ou sexual nos 12 meses que precederam o homicídio e perpetrador da violência.

Todos os parentes das vítimas incluídas neste estudo, responsáveis pela reclamação do corpo no IML, foram convidados a participar da pesquisa, sendo devidamente esclarecidos sobre o estudo e, em caso de concordância, assinaram o Termo de Consentimento Livre e Esclarecido. Nos casos em que houve recusa dos familiares em participar da pesquisa, foram registrados apenas os dados da DO. Para arquivamento e análise dos dados, utilizou-se o programa Epi Info 3.5.3 (Centers for Disease Control and Prevention, Atlanta, Estados Unidos).

\section{Resultados}

De um total de 58 casos incluídos no estudo, encontrou-se que a idade variou entre 12 e 48 anos, com media de 27 anos ( $\mathrm{DP}=8,7$ ). A maioria era adulta e de cor parda e preta. Encontrou-se maior freqüência de vítimas solteiras, com escolaridade entre um e sete anos completos, ocupação não remunerada por estarem desempregadas ou serem estudantes ou domésticas no seu lar; $31 \%$ tinham ocupação remunerada por exercício de trabalho formal ou informal (Tabela 1).

Em relação à religião, 39,7\% eram católicas, $17,2 \%$ eram evangélicas e $36,2 \%$ das informações não foram obtidas. Em $29,3 \%$ dos casos, as mulheres exerciam sua prática religiosa. A renda mensal das vítimas mostrou que $41,4 \%$ não tinham renda ou recebiam valor inferior a um salário mínimo e que $24,1 \%$ recebiam entre um e dois salários mínimos. Foi de 17,2\% a frequência de mulheres que tiveram filhos no último ano e duas as que estavam grávidas quando foram assassinadas (Tabela 1).

Em relação às características individuais de risco das vítimas, 39,7\% eram tabagistas, 48,3\% 
Características biológicas e sociodemográficas das mulheres vítimas de homicídio. Recife, Pernambuco, Brasil, 2009/2010.

\begin{tabular}{|c|c|c|}
\hline Características & $\mathrm{n}$ & $\%$ \\
\hline \multicolumn{3}{|l|}{ Idade (anos) } \\
\hline $12-19$ & 14 & 24,1 \\
\hline $20-49$ & 44 & 75,9 \\
\hline \multicolumn{3}{|l|}{ Cor } \\
\hline Branca & 9 & 15,5 \\
\hline Preta & 2 & 3,4 \\
\hline Parda & 44 & 75,9 \\
\hline Ignorado/Não informado & 3 & 5,2 \\
\hline \multicolumn{3}{|l|}{ Estado civil } \\
\hline Solteira & 50 & 86,2 \\
\hline Casada & 3 & 5,2 \\
\hline Ignorado/Não informado & 5 & 8,6 \\
\hline \multicolumn{3}{|l|}{ Escolaridade (anos de estudo) } \\
\hline $1-7$ & 34 & 58,6 \\
\hline$\geq 8$ & 18 & 31,0 \\
\hline Ignorado/Não informado & 6 & 10,3 \\
\hline \multicolumn{3}{|l|}{ Ocupação } \\
\hline Trabalho remunerado & 18 & 31,0 \\
\hline Sem remuneração & 31 & 53,4 \\
\hline Ignorado/Não informado & 9 & 15,5 \\
\hline \multicolumn{3}{|l|}{ Religião } \\
\hline Católica & 23 & 39,7 \\
\hline Evangélica & 10 & 17,2 \\
\hline Outra & 1 & 1,7 \\
\hline Sem religião & 3 & 5,2 \\
\hline Ignorado/Não informado & 21 & 36,2 \\
\hline \multicolumn{3}{|l|}{ Prática de religião } \\
\hline Sim & 17 & 29,3 \\
\hline Não & 22 & 37,9 \\
\hline Ignorado/Não informado & 19 & 32,8 \\
\hline \multicolumn{3}{|c|}{ Renda mensal da vítima (salários mínimos) } \\
\hline$>1$ & 24 & 41,4 \\
\hline $1-2$ & 14 & 24,1 \\
\hline $2-5$ & 3 & 5,2 \\
\hline Ignorado/Não informado & 13 & 29,3 \\
\hline \multicolumn{3}{|c|}{ Nascimento de filho no último ano } \\
\hline $\operatorname{Sim}$ & 10 & 17,2 \\
\hline Não & 32 & 55,2 \\
\hline Ignorado/Não informado & 16 & 27,6 \\
\hline \multicolumn{3}{|c|}{ Gravidez por ocasião do homicídio } \\
\hline Sim & 2 & 3,4 \\
\hline Não & 44 & 75,9 \\
\hline Ignorado/Não informado & 12 & 20,7 \\
\hline
\end{tabular}

Fonte: Instituto Médico Legal, 2009/2010.

faziam uso de bebidas alcoólicas, 24,1\% usavam algum tipo de droga e 10,3\% tinham algum tipo de envolvimento com o tráfico de drogas; $15,5 \%$ haviam tido passagem pela polícia. O total de vítimas com história de violência física ou sexual sofrida nos 12 meses anteriores ao homicídio foi 
de 29,3\%, e esta violência foi perpetrada pelo parceiro íntimo em $64,7 \%$ dos casos (Tabela 2 ).

Os instrumentos utilizados para o homicídio foram arma de fogo em $69 \%$ dos casos; arma branca, em 15,5\%; morte por estrangulamento, em $1,7 \%$.

\section{Discussão}

Este estudo encontrou predominância de homicídios entre mulheres adultas e de cor parda, o que confirma a prevalência da violência exercida

Tabela 2

Características de risco das mulheres vítimas de homicídio e características relacionais entre elas e o perpetrador da violência pregressa. Recife, Pernambuco, Brasil, 2009-2010.

\begin{tabular}{|c|c|c|}
\hline Características & $\mathbf{n}$ & $\%$ \\
\hline \multicolumn{3}{|l|}{ Tabagismo } \\
\hline Sim & 23 & 39,7 \\
\hline Não & 20 & 34,5 \\
\hline Ignorado/Não informado & 15 & 25,9 \\
\hline \multicolumn{3}{|l|}{ Uso de bebida alcoólica } \\
\hline Sim & 28 & 48,3 \\
\hline Não & 18 & 31,0 \\
\hline Ignorado/Não informado & 12 & 20,7 \\
\hline \multicolumn{3}{|l|}{ Uso de droga } \\
\hline Sim & 14 & 24,1 \\
\hline Não & 29 & 50,0 \\
\hline Ignorado/Não informado & 15 & 25,9 \\
\hline \multicolumn{3}{|l|}{ Envolvimento com tráfico de drogas } \\
\hline Sim & 6 & 10,3 \\
\hline Não & 31 & 53,4 \\
\hline Ignorado/Não informado & 21 & 36,2 \\
\hline \multicolumn{3}{|l|}{ Uso de arma } \\
\hline Sim & 1 & 1,7 \\
\hline Não & 43 & 75,9 \\
\hline Ignorado/Não informado & 14 & 24,1 \\
\hline \multicolumn{3}{|l|}{ Passagem pela polícia } \\
\hline $\operatorname{Sim}$ & 9 & 15,5 \\
\hline Não & 30 & 51,7 \\
\hline Ignorado/Não informado & 19 & 32,8 \\
\hline \multicolumn{3}{|l|}{ Vítima de violência física ou sexual } \\
\hline Sim & 17 & 29,3 \\
\hline Não & 24 & 41,4 \\
\hline Ignorado/Não informado & 17 & 29,3 \\
\hline \multicolumn{3}{|l|}{ Perpetrador da violência pregressa } \\
\hline Namorado ou companheiro & 11 & 64,7 \\
\hline Familiar residente no domicílio & 1 & 1.7 \\
\hline Outra pessoa & 3 & 5,2 \\
\hline
\end{tabular}

Fonte: Instituto Médico Legal, 2009/2010. contra mulheres afrodescendentes. Resultados de um estudo multicêntrico ${ }^{12}$, de um de base populacional que incluiu 15 capitais brasileiras e o Distrito Federal 13 e de outro em serviço terciário de saúde no nordeste brasileiro 14 demonstraram elevadas prevalências de violência contra a mulher. Os nossos achados assemelham-se aos encontrados em Boston (Estados Unidos) 15 e no Brasil 16, que evidenciaram maior tendência de cor negra entre as vítimas de homicídio. Também foi relevante a discriminação racial e de gênero encontrada em mulheres vítimas de violência, adultas e negras da cidade do Recife 5 . No Brasil, o não reconhecimento da desiguadade racial é uma importante causa de discriminação, que promove mais desigualdades e consequente redução de oportunidades para escolarização e de chances de ingresso no mercado de trabalho 17.

Verificou-se elevada frequência de baixa escolaridade, ocupação não remunerada e renda inferior a uma salário minímo, características reconhecidas como de risco para sofrer violência não letal e letal 6,9,13. Ademais, em conjunto, essas características podem ser extremamente danosas à subjetividade do sujeito, promovendo a insatisfação pessoal e a redução da autoestima, aumentando não só a vulnerabilidade da mulher na relação social e de gênero, como também sua adesão ao uso de bebidas alcoólicas e de outras drogas. Tais adesões apresentaram elevada frequência no presente estudo e, embora sejam comportamentos motivados inicialmente apenas pela busca da satisfação, transformam-se quase sempre em hábito ou dependência, prejudicando o desempenho adequado das tarefas do cotidiano e as relações sociais, tornando-se também maior risco para morte 18 .

Considera-se uma limitação deste estudo, e possível viés de informação, a elevada frequência de dados não obtidos, levando-se em conta as fontes e as circunstâncias da coleta de dados. No entanto, entrevistar os familiares possibilitou a inclusão de variáveis de análise, que seriam inexequíveis se o estudo fosse restrito aos dados das DOs.

A condição plural da violência requer o aprofundamento do conhecimento da população vitimada, do agressor, das circunstâncias e motivações para perpetrar o ato violento. Sugere-se a realização de outros estudos que possam lançar luz sobre as questões acerca dos homicídios femininos e melhor subsidiar medidas de enfrentamento a esta grave e importante causa externa de morte. 


\section{Resumen}

Este estudio investigó el perfil epidemiológico de mujeres víctimas de homicidio en Recife, Pernambuco, nordeste de Brasil. Se realizó un estudio observacional, descriptivo y prospectivo que incluyó a todas las mujeres de 10-49 años, víctimas de homicidios y residentes en Recife, desde marzo de 2009 hasta febrero de 2010. El instrumento utilizado fue un cuestionario de investigación de los factores socioeconómicos, demográficos, biológicos y de riesgo. Se entrevistó a los familiares de las víctimas y se recopiló datos sobre los certificados de defunción. Sesenta homicidios fueron identificados durante el período de estudio. La mayoría de las víctimas eran adultas, mulatas, un con bajo nivel de educación y con bajos ingresos, 39, 7\% eran fumadores, el consumo de alcohol y drogas ilícitas eran de un $48,3 \%$ y un $24,1 \%$, respectivamente, además un $29,3 \%$ había experimentado violencia física y/o sexual en los 12 meses anteriores al homicidio. Las armas de fuego se utilizaron en $69,0 \%$ de los casos.

Homicidio; Violencia; Mujeres

\section{Colaboradores}

M. A. Silva, J. E. Cabral Filho, M. M. R. Amorim e G. H. Falbo Neto contribuíram em todas as etapas.

\section{Agradecimentos}

Ao Fundo de Apoio à Pesquisa do Instituto de Medicina Integral Prof. Fernando Figueira (FAPE/IMIP), pelo financiamento. Ao Instituto Médico Legal Prof. Antônio Persivo Cunha/Secretaria de Defesa Social de Pernambuco.

\section{Referências}

1. Palma-Solis M, Vives-Cases C, Alvarez-Dardet C. Gender progress and government expenditure as determinants of femicide. Ann Epidemiol 2008; 18:322-9.

2. Reza A, Mercy JA, Krug E. Epidemiology of violent deaths in the world. Inj Prev 2001; 7:102-4.

3. Meetoo V, Mirza HS. There is nothing "honourable" about honour killings: gender, violence and the limits of multiculturalism. Womens Stud Int Forum 2007; 30:187-200.

4. Arnold MW, Silva MA, Falbo Neto GH, Haimenis RP. Anos potenciais de vida perdidos por mulheres em idade fértil na cidade do Recife, Pernambuco, vítimas de morte por homicídio nos anos de 2001 e 2002. Rev Bras Saúde Matern Infant 2007; 7 Suppl 1:23-7.

5. Santos SM, Barreto-Araújo TV, Guimarães MJ. Desigualdades raciais na mortalidade de mulheres adultas no Recife. Saúde Soc 2007; 16:87-102.
6. Faúndes A, Parpinelli MA, Cecatti JG. Mortalidade de mulheres em idade fértil em Campinas, São Paulo (1985-1994). Cad Saúde Pública 2000; 16:671-9.

7. Meneghel SN, Hirakata VN. Femicídios: homicídios femininos no Brasil. Rev Saúde Pública 2011; 45:564-74.

8. Departamento de Análise e Situação de Saúde, Secretaria de Vigilância em Saúde, Ministério da Saúde. Saúde Brasil 2008: uma análise da situação de saúde. Brasília: Ministério da Saúde; 2010.

9. Krug EG, Dahlberg LL, Mercy JA, Zwi A, LozanoAscencio A. Rapport mondial sur la violence et la santé. Geneva: World Health Organization; 2002.

10. Kanh T. Os custos da violência: quanto se gasta ou deixa de ganhar por causa do crime no Estado de São Paulo. São Paulo Perspect 1999; 13:42-8. 
11. Departamento de Análise e Situação de Saúde, Secretaria de Vigilância em Saúde, Ministério da Saúde. Saúde Brasil 2006: uma análise da situação de saúde. Brasília: Ministério da Saúde; 2006.

12. Schraiber LB, D'Oliveira AFP, França-Junior I, Diniz S, Portella AP, Ludermir AB. Prevalência da violência contra a mulher por parceiro íntimo em regiões do Brasil. Rev Saúde Pública 2007; 41:797-807.

13. Reichenheim ME, Moraes CL, Szklo A, Hasselmann MH, Souza ER, Lozana JA, et al. The magnitude of intimate partner violence in Brazil: portraits from 15 capital cities and the Federal District. Cad Saúde Pública 2006; 22:425-37.

14. Silva MA, Falbo Neto GH, Figueiroa JN, Cabral Filho JE. Violence against women: prevalence and associated factors in patients attending a public healthcare service in the Northeast of Brazil. Cad Saúde Pública 2010; 26:264-72.
15. Katz ME, Holmes MD, Power KL, Wise PH. Mortality rates among 15- to 44-year-old women in Boston: looking beyond reproductive status. Am J Public Health 1995; 85:1135-8.

16. Filho AMS, Souza MFM, Gazal-Carvalho C, Malta DC, Alencar AP, Silva MMA. Análise da mortalidade por homicídios no Brasil. Epidemiol Serv Saúde 2007; 16:7-18.

17. Botega NJ, Mitsuushi GN, Azevedo RCS, Lima DD, Fanger PC, Mauro MLF. Depression, alcohol use disorders and nicotine dependence among patients at a general hospital. Rev Bras Psiquiatr 2010; 32:250-6.

18. Gawryszewski VP, Kahn T, Mello-Jorge MHP. Informações sobre homicídios e sua integração com o setor saúde e segurança pública. Rev Saúde Pública $2005 ; 39: 627-33$.

Recebido em 14/Dez/2011

Versão final reapresentada em 19/Fev/2012

Aprovado em 13/Nov/2012 